\title{
Halloysite nanotube with fluorinated lumen: Non-foaming nanocontainer for storage and controlled release of oxygen in aqueous media
}

\author{
Giuseppe Cavallaro, Giuseppe Lazzara*, Stefana Milioto, Giovanni Palmisano, Filippo Parisi \\ Department of Physics and Chemistry, Università degli Studi di Palermo, Viale delle Scienze, pad. 17, 90128 Palermo, Italy
}

\section{A R T I C L E I N F O}

\section{Article history:}

Received 9 October 2013

Accepted 10 November 2013

Available online 20 November 2013

\section{Keywords:}

Halloysite

Nanoclay

Fluorinated surfactant

Gas solubilization

\begin{abstract}
A B S T R A C T
Halloysite clay nanotubes were selectivity modified by adsorbing perfluoroalkylated anionic surfactants at the inner surface. The modified nanotubes formed kinetically stable dispersions due to the enhanced electrostatic repulsions exercised between the particles. We proved that the modified nanotubes can be used as non-foaming oxygen nanocontainers in aqueous media. The gas release from supersaturated dispersions can be controlled by external stimuli and system composition. In conclusion, we managed to put forward an easy strategy to develop smart materials from natural nanoclays, which can endow important applications like the storage and delivery of gas.
\end{abstract}

(c) 2013 Elsevier Inc. All rights reserved.

\section{Introduction}

Covalent or non-covalent nanoparticle modification is a well known strategy to design new target properties. Sustainable nanoparticles are represented by clays available with different shapes, sizes and surface chemical properties. Most common clay nanoparticles possess a nanolayered morphology, such as kaolin and montmorillonite. Notwithstanding, there are natural nanoclays with hollow tubular shape [1,2] of great scientific interest due to potential applications. Promising nanoclays as nanocontainers with controlled release properties are imogolite [1] and natural halloysite (HNT) [2]. Besides the interesting properties, imogolite presents a certain toxicity [3]. Halloysite mineral is well known [4] since 1946 but its application in smart sustainable materials has been proposed only a few years ago [2]. HNT is abundant, durable and biocompatible and, furthermore, it is cheap compared to synthetic nanomaterials with similar morphology. HNT is generated by rolling-up a kaolin sheet with a still unknown mechanism; its size ranges between 0.5 and $1 \mu \mathrm{m}$ in length and between 15 and $100 \mathrm{~nm}$ in the inner diameters [2].

The different chemistry of the inner and the outer surfaces makes HNT a tremendous tool. Being that the external surface is composed of $\mathrm{Si}-\mathrm{O}-\mathrm{Si}$ groups and the internal surface of a gibbsite-like array of $\mathrm{Al}-\mathrm{OH}$ groups, the aqueous acid-base equilibria confer negative and positive charges, at the outer and inner surfaces respectively, in a wide $\mathrm{pH}$ range $[5,6]$. Consequently, selective adsorption of ionic

\footnotetext{
* Corresponding author.

E-mail address: giuseppe.lazzara@unipa.it (G. Lazzara).
}

species may strategically control the charge. The hydrophobic modification of HNT lumen with anionic surfactant stabilized successfully nanotubes in water [7] as a consequence of canceling the positive charges at the surface due to the entrapment of the anionic molecule into the HNT lumen generating a large net negative charge. Nanotube applications have been proposed for controlled release in healing anticorrosion [8,9], water purification [10], polymer composites [11,12] and antimicrobial coatings [13]. The covalent modification of the inner surface generated HNT able to incorporate more ferrocene than pristine nanoclay [14]. To the light of the phase behaviors of HNT dispersion, those nanoparticles are considered strategic for fabrication of long-range ordered nano-objects [15]. Thus, development of tube-like materials with tunable properties is one of the most scientific challenges.

We report the modification of HNT lumen by incorporating perfluoroalkylated anionic surfactants. This was done with the intent at obtaining rather stable colloidal dispersions in water with enhanced oxygen solubilization ability. Fluorinates are well established for chemical and biological inertness, fire extinguishing and flame retardant materials [16]. Finally, fluorinated biocompatible surfactants have been proposed as oxygen carriers in biomedical applications [17].

\section{Experimental section}

\subsection{Materials}

Halloysite nanotubes (HNTs) and kaolin are Sigma products. Perfluorooctanoic acid (PFC8H), Perfluoroheptanoic acid (PFC7H) 
and Perfluoropentanoic acid (PFC5H) from Fluka were crystallized from carbon tetrachloride and dried at room temperature. Their sodium salts (NaPFC8, NaPFC7 and NaPFC5) were prepared by neutralization with an aqueous sodium hydroxide solution. The products were crystallized twice from an ice-cold solution and dried in a vacuum oven at $60^{\circ} \mathrm{C}$ for at least 4 days before their use. The structure of the surfactants is given in Chart 8 .

Water from reverse osmosis (Elga model Option 3) with a specific resistivity greater than $1 \mathrm{M} \Omega \mathrm{cm}$ was used.

\subsection{Preparation of surfactants functionalized HNTs}

To aqueous surfactant solutions $\left(0.1 \mathrm{~mol} \mathrm{~kg}^{-1}\right) 0.02 \mathrm{~g} \mathrm{~cm}^{-3}$ of HNTs was added. The obtained dispersions were magnetically stirred for ca. 1 day. Afterward, they were taken in a vacuum oven at $50{ }^{\circ} \mathrm{C}$ and at 100 mbar overnight. The solid was separated by centrifugation and rinsed several times with water until the surface tension of the supernatant was close to the value for pure water (ca. $72 \mathrm{mN} \mathrm{m}^{-1}$ ). This ensures the absence of unbound surfactant. All of the solids dried at $80^{\circ} \mathrm{C}$ were investigated by means of thermogravimetric analysis (TG) to estimate the surfactant loading into the HNT lumen.

\subsection{Methods}

$\zeta$-potential and dynamic light scattering (DLS) measurements were carried out by means of a Zetasizer NANO-ZS (Malvern Instruments). The field-time autocorrelation functions were well described by a single decay, which provides the decay rate $(\Gamma)$ of the diffusive mode. For the translational motion, the collective diffusion coefficient at a given concentration is $D_{\mathrm{t}}=\Gamma / q^{2}$ where $\mathrm{q}$ is the scattering vector given by $4 \pi n \lambda^{-1} \sin (\theta / 2)$ being $\mathrm{n}$ the water refractive index, $\lambda$ the wavelength $(632.8 \mathrm{~nm})$ and $\theta$ the scattering angle $\left(173^{\circ}\right)$.

The functionalized nanotubes were imaged by using a microscope ESEM FEI QUANTA 200F. The measurements were carried out in high vacuum mode $\left(<6 \times 10^{-4} \mathrm{~Pa}\right)$ for simultaneous secondary electron, the energy of the beam was $30 \mathrm{kV}$ and the working distance was $10 \mathrm{~mm}$. Before each experiment, the sample was coated with gold in argon by means of an Edwards Sputter Coater S150A to avoid charging under electron beam.

The thermogravimetric analyses were done by using a Q5000 IR apparatus (TA Instruments) under the nitrogen flow of $25 \mathrm{~cm}^{3}$ $\mathrm{min}^{-1}$ for the sample and $10 \mathrm{~cm}^{3} \mathrm{~min}^{-1}$ for the balance at the heating rate of $10^{\circ} \mathrm{C} \mathrm{min}{ }^{-1}$. Temperature spanned from ambient to $900{ }^{\circ} \mathrm{C}$. The surfactant:HNT ratio was determined from the residual mass by taking into account for the water content as reported in the literature $[16,12]$.

The densities $\left( \pm 1 \times 10^{-6} \mathrm{~g} \mathrm{~cm}^{-3}\right)$ and speed of sound $\left( \pm 0.1 \mathrm{~m} \mathrm{~s}^{-1}\right)$ of the liquid dispersion were determined at 25.000 $\pm 0.001{ }^{\circ} \mathrm{C}$ by using a density and sound velocity meter (DSA $5000 \mathrm{M}$, Anton Paar).

The specific volume of pristine or modified HNTs $\left(v_{\mathrm{sp}}\right)$ was calculated as follows

$v_{\mathrm{sp}}=1 / d-10^{2}\left(d-d_{0}\right) /\left(C_{\mathrm{S}} \times d \times d_{0}\right)$

where $d$ and $d_{0}$ are the dispersion and water densities, respectively; $C_{S}$ is the concentration of solid material into the dispersion in $\mathrm{g} /$ $100 \mathrm{~g}$ of solvent. The isentropic compressibility coefficients of the dispersions $(\beta)$ were obtained as $100 /\left(u^{2} \times d\right)$ being $u$ the ultrasonic velocity of the dispersion. The specific isentropic compressibility $\left(k_{\mathrm{s}}\right)$ was calculated using the following equation.

$k_{\mathrm{s}}=v_{\mathrm{sp}} \beta+10^{2}\left(\beta-\beta_{0}\right) /\left(C_{\mathrm{S}} \times d_{0}\right)$ where $\beta_{0}$ is the isentropic compressibility coefficients of water and the other symbols have the same meaning as above.

The sedimentation volume of pristine and modified HNTs was determined by using tubes of borosilicate glass with an inner diameter of ca. $2.3 \mathrm{~mm}$ and length of ca. $125 \mathrm{~mm}$. The tubes were filled with the dispersion and left to equilibrate in vertical position. Two phases were observed, an upper transparent phase and a lower milk-like phase. The transparent upper phase is water according to the density value. On this basis, the concentration of either HNTs or functionalized HNTs in the lower phase $\left(C_{\mathrm{LP}}\right)$ was determined as $C_{\mathrm{LP}}=C_{\mathrm{S}} / R_{\mathrm{LP}} \mathrm{T}$, where $C_{\mathrm{S}}$ is the stoichiometric initial concentration of material in water, $R_{\mathrm{LP}: \mathrm{T}}$ is the $V_{\mathrm{LP}} / V_{\mathrm{T}}$ ratio being $V_{\mathrm{T}}$ and $V_{\mathrm{LP}}$ the total and the lower phase volumes, respectively. The tubes were imaged and $\mathrm{R}_{\mathrm{LP}: \mathrm{T}}$ was estimated from the height of the meniscus in the capillary by using an image analyzer software (Image $1.43 \mathrm{u}$ ).

The oxygen desaturation curves were obtained by using a HD22559.2 apparatus (Delta Ohm). Water, HNT and modified HNT aqueous dispersions (ca. $15 \mathrm{~cm}^{3}$ ) were saturated with oxygen by bubbling the pure gas for ca. $1 \mathrm{~h}$. The oxygen concentration was registered every $2 \mathrm{~min}$. The experiments were carried out under static conditions and under magnetic stirring at $1250 \mathrm{rpm}$. The oxygen concentration was normalized for the solubility of the gas in water, $\left[\mathrm{O}_{2}\right]_{\text {sat }}$, at the experimental conditions $\left(25^{\circ} \mathrm{C}\right.$ and $1 \mathrm{~atm})$.

\section{Results and discussion}

The alumina inner surface of the nanotube was selectively modified with perfluorinated anionic surfactants rendering the total charge of the nanotube significantly changed. The as prepared hybrid nanotubes exhibit an enhancement of stability in water and a core to successfully entrap oxygen in aqueous media.

\subsection{Physico-chemical characterization of functionalized Halloysite nanotubes}

The amount of surfactant adsorbed onto the HNT (Table 1) was estimated from the thermogravimetric analysis from the residual mass by taking into account for the water content as reported in the literature $[16,12]$. These values are far below the maximum loading ability of HNTs being that the hollow cavity represents ca. $10 \%$ of the nanoclay volume. By assuming an adsorbed surfactant monolayer and considering $760 \mathrm{~m}^{2} \mathrm{~g}^{-1}$ for the occupied area of NaPFC8 at the alumina/water interface [19], one calculates the surfactant loading of $0.90 \mathrm{wt} \%$ that is in straightforward agreement with the experimental value (Table 1 ). These calculations cannot be extended to NaPFC7 and NaPFC5 because, to the best of our knowledge, no adsorption data at the alumina/water interface are available; nevertheless, it is expected a behavior rather comparable in terms of area per molecule if an extended chain configuration is considered [19]. From experimental loading data one can conclude that NaPFC7 and NaPFC5 did not fully cover the HNT lumen.

Table 1

Surfactant loading, diffusion coefficient and $\zeta$-potential for HNT/surfactant hybrid materials ${ }^{\mathrm{a}}$

\begin{tabular}{llll}
\hline & Surfactant loading & $D^{\circ} \times 10^{12}$ & $\zeta$-potential \\
\hline HNT & & $1.5 ; 0.94^{\mathrm{b}}$ & $-21 ;-19.6^{\mathrm{b}}$ \\
HNT/NaPFC5 & 0.29 & 1.2 & -27 \\
HNT/NaPFC7 & 0.62 & 1.0 & -29 \\
HNT/NaPFC8 & 0.86 & 0.90 & -32
\end{tabular}

a Units are: surfactant loading, wt $\% ; D^{\circ}, \mathrm{m}^{2} \mathrm{~s}^{-1} ; \zeta, \mathrm{mV}$. Errors are: surfactant loading, $4 \% ; D^{\circ}, 5 \% ; \zeta, 4 \%$.

b From Ref. [7]. 

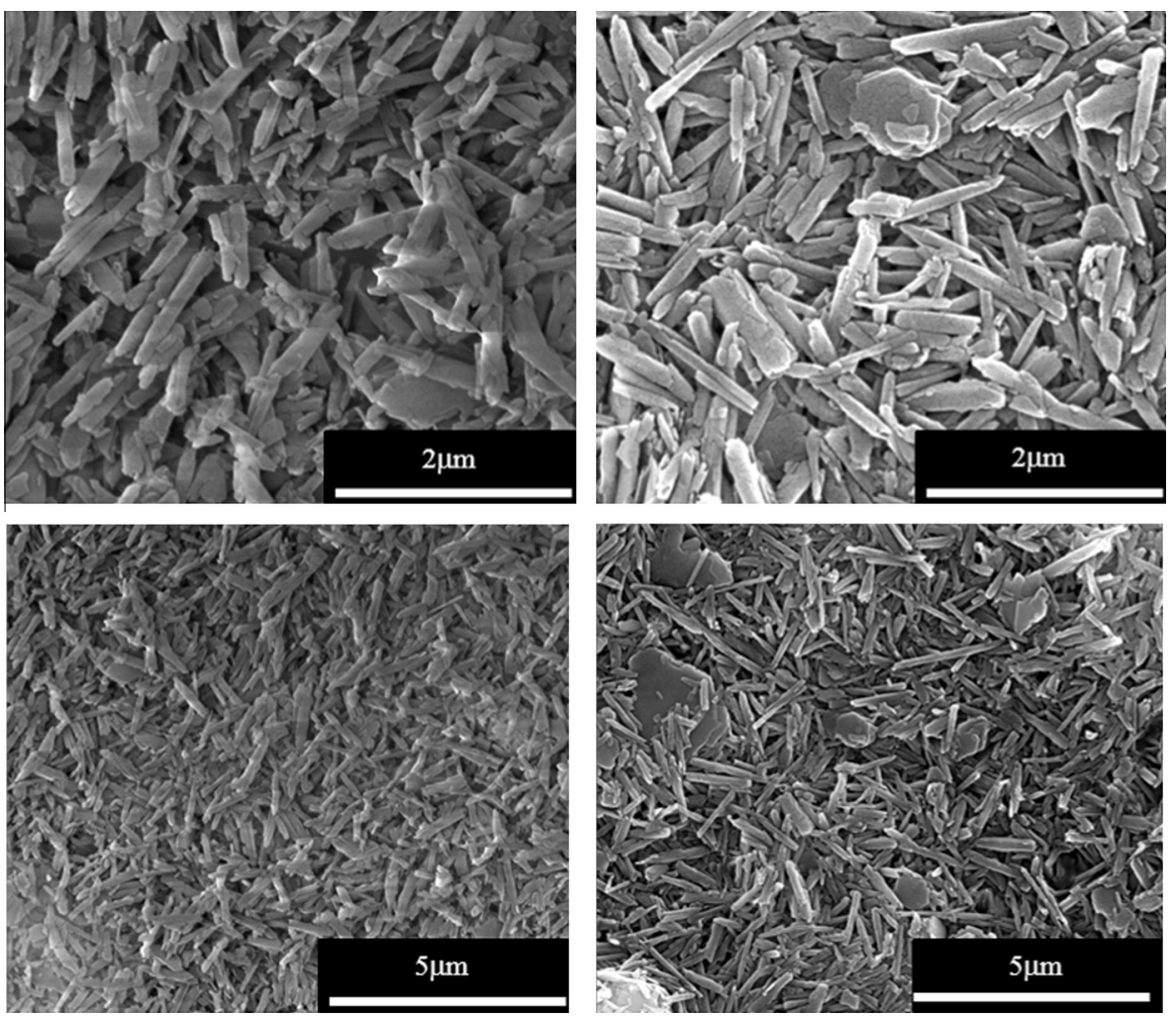

Fig. 1. Scanning electron micrographs of HNT/NaPFC5 (left hand side) and HNT/NaPFC8 (right hand side).

The SEM micrographs (Fig. 1) showed that the tubular shape of halloysite is preserved after the adsorption of perfluorinated surfactants and the characteristic lengths are comparable to those observed for the pristine HNT samples in agreement with the monolayer formation [18]. The dried samples did not show any orientation of the anisotropic particles ruling out preferential interactions. This aspect is fundamental as far as the aqueous dispersions stability is concerned.

To shed more light to this aspect, the translational diffusion coefficient and the charge of the hybrid materials were determined in dilute aqueous dispersions. The measurements were carried out at various contents of the dispersed material but the concentration effect was negligible. The diffusion coefficient $\left(D^{\circ}\right)$ and the $\zeta$-potential $(\zeta)$ extrapolated at infinite dilution are reported in Table 1 . The data show that the hybrid materials did not aggregate in water and they diffuse as single nanotubes. The $\zeta$-potential data (Table 1) indicate that the net negative charge of the nanotubes is raised up by the surfactant adsorption in agreement with the neutralization of the positive charges of the inner surface.

To definitely rule out the presence of aggregates and to evidence eventual particle-particle interactions, the specific volume $\left(v_{\mathrm{sp}}\right)$ and the isoentropic compressibility $\left(k_{\mathrm{s}}\right)$ of HNT and HNT/NaPFC8 dispersed in water were determined (Fig. 2). Both $v_{\mathrm{sp}}$ and $\mathrm{k}_{\mathrm{s}}$ are linearly dependent on the concentration of the material and can be fitted according to the Mc-Millan-Mayer approach

$v_{\mathrm{sp}}=v^{\circ}+B_{\mathrm{v}} C_{\mathrm{s}} \quad k_{\mathrm{s}}=k^{\circ}+B_{\mathrm{k}} C_{\mathrm{S}}$

where $v^{\circ}$ and $k^{\circ}$ are the specific volume and compressibility at infinite dilution, respectively, while $B_{\mathrm{v}}$ and $B_{\mathrm{k}}$ are the particle-particle interaction parameters for volume and compressibility, respectively. Within the experimental errors, the properties extrapolated at infinite dilution do not reflect differences between HNT and the hybrid material (Table 2). By using the surfactant loading from Table 1 , the $v^{\circ}$ value for NaPFC $8\left(0.458 \mathrm{~cm}^{3} \mathrm{~g}^{-1}\right)$ [20] and HNT, one can

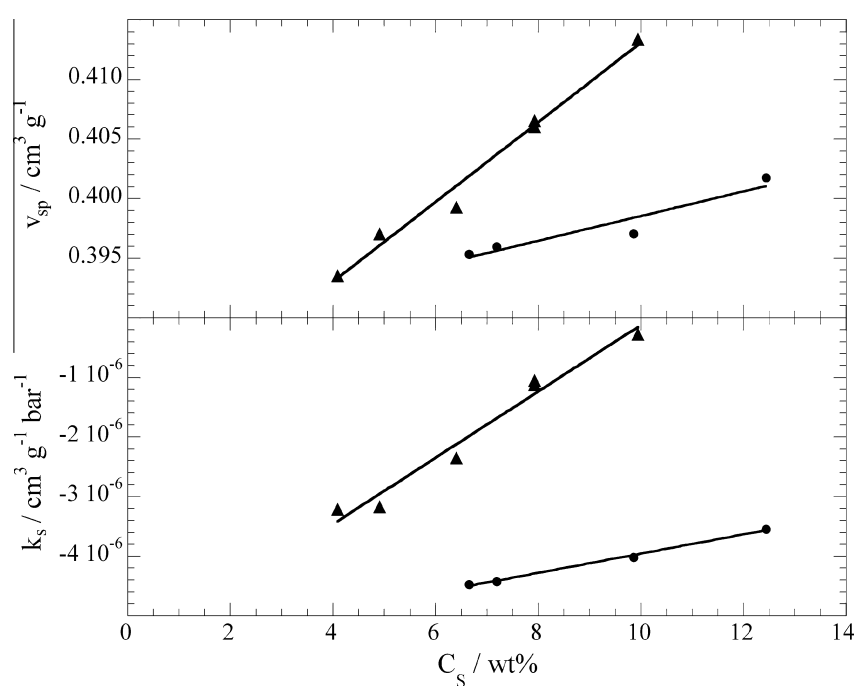

Fig. 2. Specific volume and isoentropic compressibility for aqueous dispersion of HNT $(\bullet)$ and HNT/NaPFC8 hybrid materials $(\boldsymbol{\Delta})$ as a function of concentration.

Table 2

Volume and compressibility data ${ }^{\mathrm{a}}$.

\begin{tabular}{lll}
\hline & HNT & HNT/NaPFC8 \\
\hline$v^{\circ}$ & $0.388 \pm 0.002$ & $0.380 \pm 0.002$ \\
$B_{\mathrm{v}}$ & $(1.0 \pm 0.2) \times 10^{-4}$ & $(3.3 \pm 0.2) \times 10^{-4}$ \\
$k^{\circ}$ & $-(5.57 \pm 0.07) \times 10^{-6}$ & $-(5.7 \pm 0.3) \times 10^{-6}$ \\
$B_{\mathrm{k}}$ & $(1.62 \pm 0.08) \times 10^{-8}$ & $(5.6 \pm 0.5) \times 10^{-8}$ \\
\hline
\end{tabular}

${ }^{\mathrm{a}}$ Units are: $v^{\circ}, \mathrm{cm}^{3} \mathrm{~g}^{-1} ; k^{\circ}, \mathrm{cm}^{3} \mathrm{~g}^{-1} \mathrm{bar}^{-1} ; B_{\mathrm{v}}, \mathrm{cm}^{3} \mathrm{~g}^{-2} \mathrm{~kg} ; B_{\mathrm{k}}, \mathrm{cm}^{3} \mathrm{~g}^{-2} \mathrm{~kg} \mathrm{bar}^{-1}$.

calculate $\mathrm{v}^{\circ}$ for the composite material by means of the rule of mixtures. As a result, the change in $v^{\circ}$ for HNT/NaPFC8 with respect to 
the value for HNT is predicted to be less than $0.1 \%$ in agreement with the experimental findings. As concerns the particle-particle interaction parameters it is reported [21] that hydrophobic forces provide negative values for $B_{v}$ while the electrostatic interactions render the water molecules less compressible [21] so that, $B_{\mathrm{v}}$ and $B_{\mathrm{k}}$ are expected to be positive. Therefore one may deduce that the nanoparticle-nanoparticle electrostatic interactions are enhanced in the hybrid material in agreement with the $\zeta$-potential results.

\subsection{Sedimentation volume of pristine and functionalized Halloysite nanotubes}

The sedimentation of the clay nanotubes assumes a key role in understanding the colloidal stability. To quantitatively explore such a phenomenon, concentrated dispersions of pristine and functionalized HNT in water were prepared and left to equilibrate in a glass tube. After 1 week, two phases were clearly identified; namely, a bottom milk-like phase and an upper transparent phase. The systems remained unaltered for 6 months at least. The lower phase is the sedimentation volume and its value increased with the initial total concentration of the material as examples shown in Fig. 3. For most concentrated samples, the upper transparent phase was not observed at all. It is noteworthy that such a peculiar sedimentation was not observed for kaolin, which possesses the same chemical composition as HNT but a sheet-like morphology. In such a case a complete sedimentation even at $8 \mathrm{wt} \%$, the maximum concentration investigated for HNT, was observed. Different morphologies confer different properties to the particles.

The sedimentation volume is a very complex parameter; nevertheless, at least in water, it is controlled by the repulsive forces exercised between particles [22]. For instance, the electrostatic repulsions are caused by the double layer surrounding each particle and the particle charge. Consequently, if the particles repel to each other they remain independent until they reach the closest packing, which will represent the concentration in the sedimentation volume. If the particles do not strongly repel to each other, they are sticking together generating a smaller sedimentation volume.

Fig. 4 shows the dependence of $R_{\mathrm{LP}: \mathrm{T}}$ on the stoichiometric concentration of the nanotubes. For all of the investigated systems, we observed a linear increase in $R_{\mathrm{LP}: \mathrm{T}}$ up to the limit of 1 , beyond which it is constant. These data indicate that the sedimentation does not take place over the concentrated regime regardless of

HNT/NaPFC5

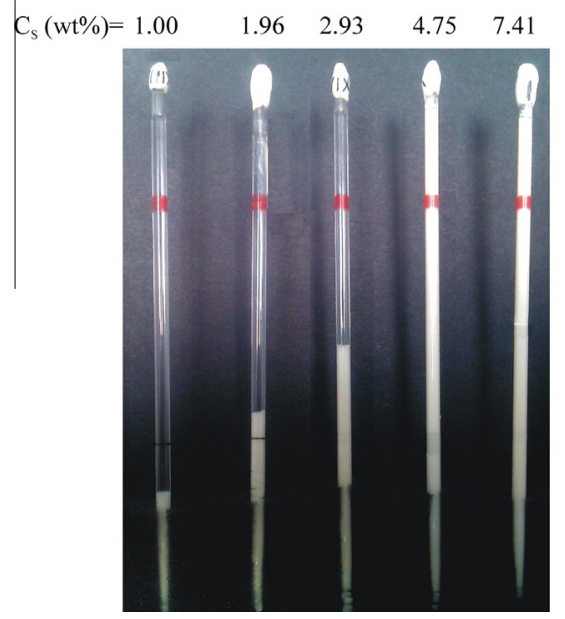

Fig. 3. Optical images of sedimentation volume in glass tubes. The tube length is $125 \mathrm{~mm}$.

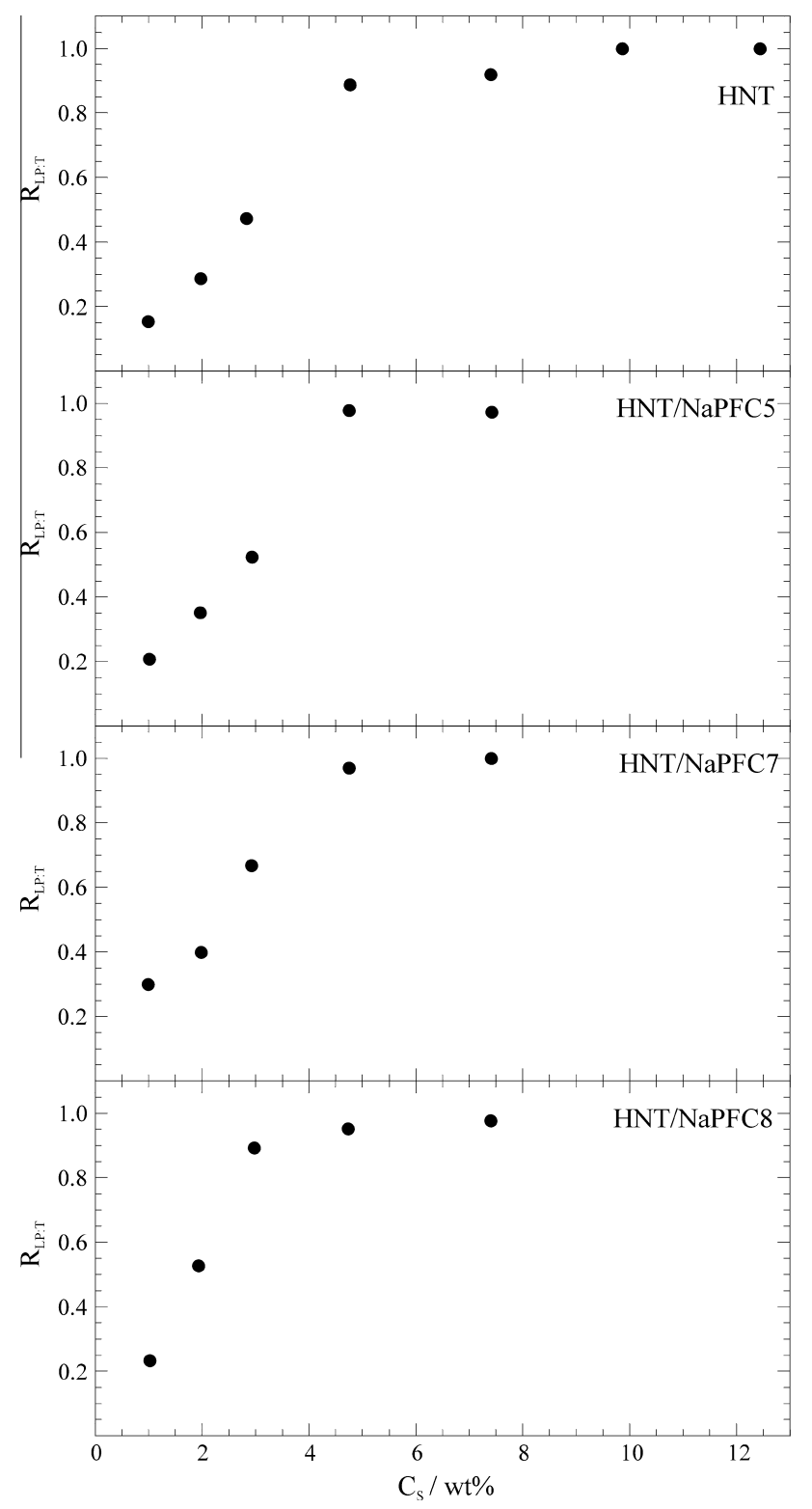

Fig. 4. Dependence of the ratio between the volume of lower phase and total volume on stoichiometric concentration of the dispersed material.

the presence and the nature of the surfactant. From the inverse of the positive slope of the linear trends shown in Fig. 4, we calculated the concentrations of the lower phase $\left(C_{\mathrm{LP}}^{*}\right)$, which are reported in Table 3. Such values are a sort of critical concentration above which the dispersion is stable because the highest packing of the nanotubes is approached and shorter distances between the nanoparticles are hindered by the electrostatic repulsions. The

Table 3

Critical concentration of the lower phase for HNT and HNT/surfactant hybrid materials in water ${ }^{\mathrm{a}}$.

\begin{tabular}{ll}
\hline & $C_{\mathrm{LP}}^{*}$ \\
\hline HNT & 6.3 \\
HNT/NaPFC5 & 5.4 \\
HNT/NaPFC7 & 4.3 \\
HNT/NaPFC8 & $3.8 ; 4.5^{\mathrm{b}}$ \\
\hline
\end{tabular}

a Units are: $C_{\mathrm{LP}}^{*}$, wt\%. Error for $C_{\mathrm{LP}}^{*}$ is less than $3 \%$.

b In $\mathrm{KCl} 0.1 \mathrm{~mol} \mathrm{~kg}^{-1}$. 
$C_{\mathrm{LP}}^{*}$ is highly altered by the presence of the anionic surfactant (Table 3) that is in agreement with the large net charge of the hybrid HNT compared to pristine HNT.

Although a quantitative interpretation of this phenomenon from a microscopic view-point might be very challenging we thought it would be interesting to compare the experimental $C_{\mathrm{LP}}^{*}$ with the computed value. This was done by assuming a simple cubic model and a contact distance given by the average length of the nanotubes (see Fig. 5). Briefly, when the concentration generates some overlapping, the rotation of each individual nanoparticle becomes restricted and the cylinders are entangled [23]. From such a geometrical model, one obtains the critical volume fraction $\varphi^{*}$ for cylinders

$\varphi^{*}=\pi R^{2} / L^{2}$

where $R$ and $L$ are the external radius and the length of the nanotubes (Fig. 5). By introducing in Eq. (4) the average outer radius $(73 \mathrm{~nm})$ and the length $(770 \mathrm{~nm})$ of HNT [18], the $\varphi^{*}$ value of 0.028 was obtained. By taking into account for the specific volume of HNT (Table 2) the $C_{\mathrm{LP}}^{*}$ value of $6.9 \mathrm{wt} \%$ was calculated. An excellent agreement between the computed and the experimental (Table 3) values of pristine nanotubes was found. On this basis one can state that the sedimentation volume of HNTs is driven by hard-cylinder interactions, and the aqueous dispersion looks stable when the contact distance between nanoparticles is approached.

As concerns the hybrid materials, $\zeta$-potential data evidenced an increase in the net charge in the modified HNT. Therefore, the particle-particle repulsive interactions shift to longer range increasing the closest average distance between the functionalized nanoparticles. This description explains the $C_{\mathrm{LP}}^{*}$ decrease for functionalized HNTs. It is noteworthy the straight correlation between $C_{\mathrm{LP}}^{*}$ and the amount of loaded surfactant into the hybrid material (Fig. 6). This reveals that each mole of adsorbed surfactant neutralizes an equivalent number of positive charges of the nanotubes inner surface generating a linear increase in the net negative charge of HNT and therefore longer range interactions. To highlight the significance of the electrostatic forces on the sedimentation behavior, $0.1 \mathrm{~mol} \mathrm{~kg}^{-1}$ of $\mathrm{KCl}$ was added to the $2.97 \mathrm{wt} \% \mathrm{NaPFC} / \mathrm{HNT}$ dispersion. The determined $C_{\mathrm{LP}}^{*}$ increases in agreement with the reduction in the mean distance between particles generated by the salt screening effect, as expected by the DLVO theory, for which higher ionic strength screens electrostatic repulsions due to the contraction of the double layer width [24].

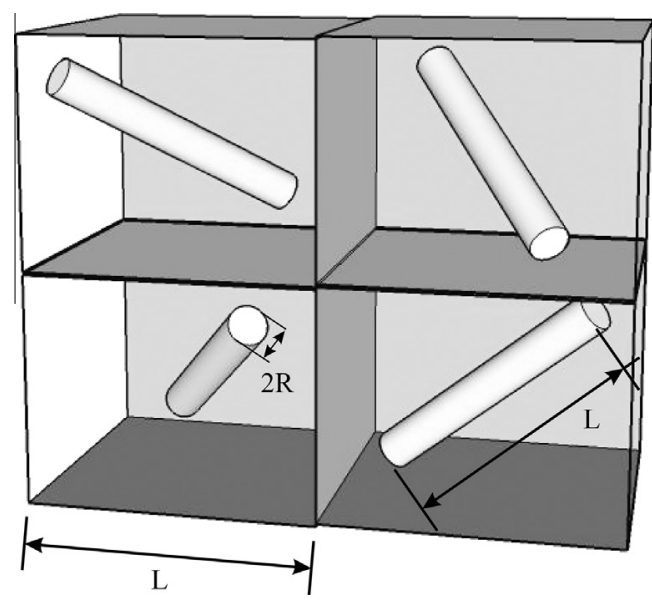

Fig. 5. Sketch representation of the simple cubic model for the interpretation of the sedimentation volume.

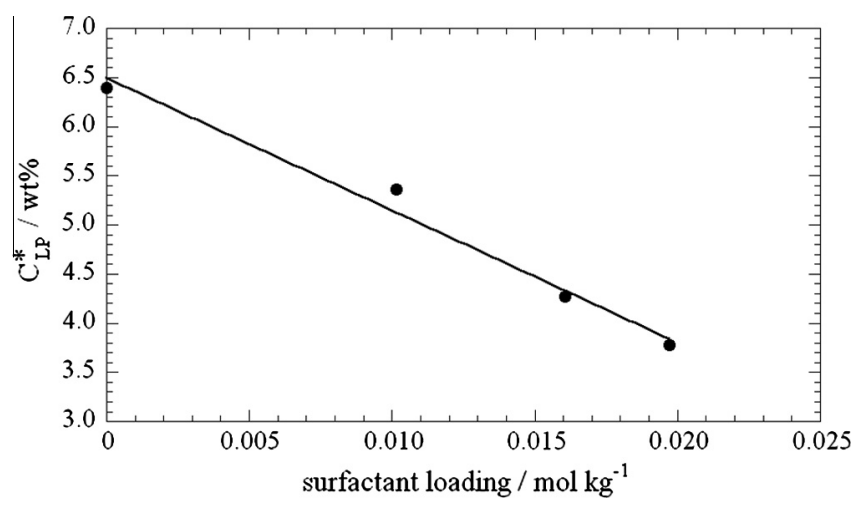

Fig. 6. Critical concentration as a function of the surfactant loading for HNT hybrid materials.

\subsection{Hybrid HNT/surfactant: non-foaming nanoreservoir for oxygen storage}

The nanotubes with highly hydrophobic cores might allow encapsulation of molecules of interest within fields like nanotechnology, drug delivery and gas storage. These prepared hybrid materials can be promising as oxygen nanoreservoir in aqueous media being that fluorinated solvents are able to solubilize large amount of gas. To explore this perspective, aqueous dispersions of both HNT and HNT/NaPFC8 (2.0 wt\%) were saturated with oxygen and left to equilibrate with air under two very different situations, i.e. vigorous stirring $(1250 \mathrm{rpm})$ and static conditions. For comparison purposes, experiments in pure water were also carried out. In case of fluorinated surfactant based HNT, foam was not generated during the gas bubbling as well as during stirring conditions in contrast to solution containing conventional fluorinated micelles. As concerns the results collected under stirring (Fig. 7A), an induction time is required before the $\mathrm{O}_{2}$ concentration starts to monotonically decrease to reach the equilibrium. The induction time follows the order water $<\mathrm{HNT}<\mathrm{HNT} /$ NaPFC8 (Table 4). Moreover, the time dependence of the $\mathrm{O}_{2}$

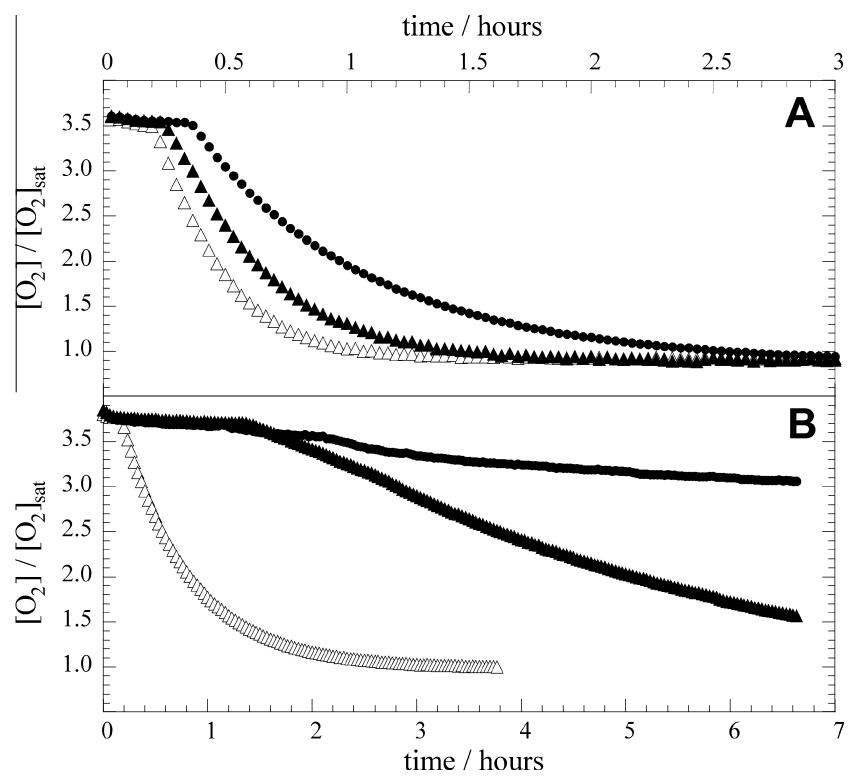

Fig. 7. Oxygen concentration as a function of time in water $(\Delta)$, in aqueous dispersions of HNT $(\boldsymbol{\Delta})$ and $\operatorname{HNT} / \operatorname{NaPFC} 8(\bullet)$. Data collected under magnetic stirring at $1250 \mathrm{rpm}(\mathrm{A})$ and under static conditions (B). 

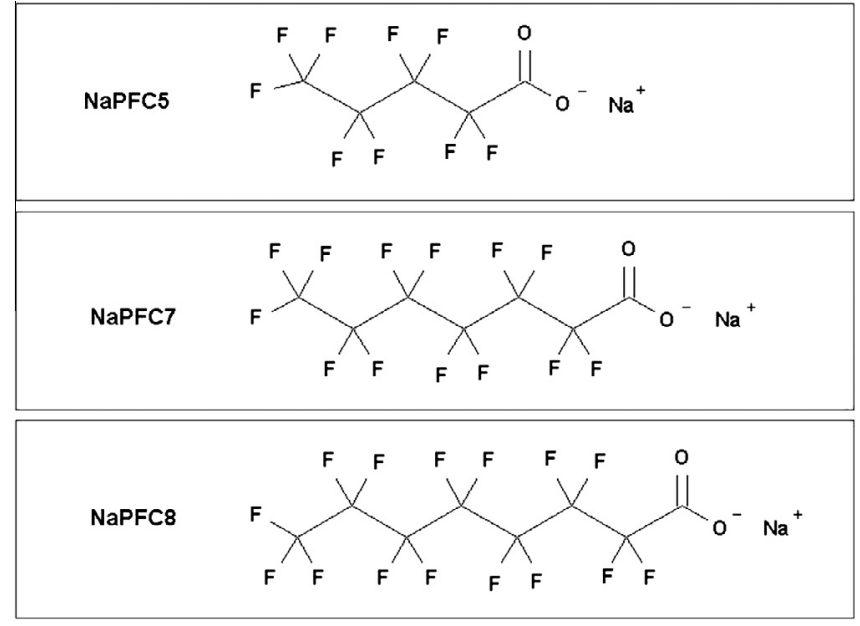

Chart 8. Structure of the fluorinated surfactants.

Table 4

Parameters for oxygen release from supersaturated systems ${ }^{\mathrm{a}}$.

\begin{tabular}{lll}
\hline & Induction time & $t_{1 / 2}$ \\
\hline Under stirring condition & & \\
Water & 12 & 31 \\
Water/HNT & 14 & 42 \\
Water/HNT/NaPFC8 & 22 & 68 \\
Under static condition & & \\
Water & 11 & 37 \\
Water/HNT & 83 & 242 \\
Water/HNT/NaPFC8 & 126 & $>400$ \\
\hline
\end{tabular}

a Units are min for induction time and $t_{1 / 2}$. Error is $\pm 2 \mathrm{~min}$.

concentration in the aqueous phase is rather smooth in the presence of HNT/NaPFC8. From the curves in Fig. 7A the time required for the $\mathrm{O}_{2}$ concentration to halve its initial value $\left(t_{1 / 2}\right)$ was calculated and reported in Table 4 . The $t_{1 / 2}$ value for HNT/NaPFC8 (twice that for pure water) shows that the hybrid material behaves like an $\mathrm{O}_{2}$ reservoir that releases the gas in water over time to contrast the desaturation of the aqueous dispersion resulting an efficient tool in retarding the $\mathrm{O}_{2}$ desaturation.

The key role played by the nanotubes on the oxygen release under static situation is impressive (Fig. 7B). The kinetics of oxygen release from water is essentially unchanged compared to the result under stirring as the $t_{1 / 2}$ values prove (Table 4 ). On the contrary, both HNT and HNT/NaPFC8 are efficient in keeping the supersaturation state. Nevertheless, after $6 \mathrm{~h}$ the HNT dispersion released ca. $50 \%$ of $\mathrm{O}_{2}$ while the hybrid nanotubes showed an oxygen loss of ca. $10 \%$. This peculiar condition is well described by both the induction and the $t_{1 / 2}$ values that follow the order water $<\mathrm{HNT} \ll \mathrm{HNT} / \mathrm{NaP}$ FC8 (Table 4). The strong enhancement of oxygen entrapment ability of the modified lumen is generated by the presence of only 0.86 wt\% of surfactant, which corresponds to the concentration value of $5 \times 10^{-5} \mathrm{~mol} \mathrm{~kg}^{-1}$ that is two order of magnitude smaller than the sodium perfluorooctanoate critical micellar concentration $\left(0.05 \mathrm{~mol} \mathrm{~kg}^{-1}\right)$ [25].

In conclusion, the presence of a fluorinated cavity generates excellent performances of the hybrid material in incorporating oxygen. In response to external stimuli (namely stirring rate) the material exhibits purposefully oxygen release.

\section{Conclusions}

We prepared hybrid nanotubes by selectively adsorbing perfluorinated anionic surfactants. These nanomaterials were highly stable (at least for six months) in water due to the key role played by the electrostatic repulsions. This kinetics stability makes them of interest for applications. The peculiar hollow tubular morphology hydrophobized with perfluorinated chains endows their use as nanocontainers to entrap apolar compounds. We proved that these nanohybrid materials are excellent reservoirs for oxygen in water and exhibit gas release under controlled situations in response to external stimuli. In conclusion, we provided an easy processing and facile surface modification to prepare sustainable and biocompatible materials promising for gas delivery applications.

\section{Acknowledgments}

The work was financially supported by the University of Palermo, PRIN 2010-2011 (prot. 2010329WPF), FIRB 2012 (prot. RBFR12ETL5) and PON 2007-2013 STI-TAM.

\section{Appendix A. Supplementary material}

Supplementary data associated with this article can be found, in the online version, at http://dx.doi.org/10.1016/j.jcis.2013.11.026.

\section{References}

[1] W. Ma, W.O. Yah, H. Otsuka, A. Takahara, J. Mater. Chem. 22 (2012) (1892) $11887-11892$.

[2] Y.M. Lvov, D.G. Shchukin, H. Mohwald, R.R. Price, ACS Nano 2 (2008) 814-820.

[3] W. Liu, P. Chaurand, C. Di Giorgio, M. De Méo, A. Thill, M. Auffan, et al., Chem. Res. Toxicol. 25 (2012) 2513-2522.

[4] D.M.C. Macewan, Nature 157 (1946) 159-160.

[5] V. Vergaro, E. Abdullayev, Y.M. Lvov, A. Zeitoun, R. Cingolani, R. Rinaldi, et al., Biomacromolecules 11 (2010) 820-826.

[6] E. Abdullayev, A. Joshi, W. Wei, Y. Zhao, Y. Lvov, ACS Nano 6 (2012) 7216-7226.

[7] G. Cavallaro, G. Lazzara, S. Milioto, J. Phys. Chem. C 116 (2012) (1938) $21932-$ 21938.

[8] E. Abdullayev, Y. Lvov, J. Mater. Chem. 20 (2010) 6681-6687.

[9] G.L. Li, Z. Zheng, H. Möhwald, D.G. Shchukin, ACS Nano 7 (2013) 2470-2478.

[10] Y. Zhao, E. Abdullayev, A. Vasiliev, Y. Lvov, J. Colloid Interface Sci. 406 (2013) 121-129.

[11] S.A. Hashemifard, A.F. Ismail, T. Matsuura, J. Colloid Interface Sci. 359 (2011) 359-370.

[12] G. Cavallaro, D.I. Donato, G. Lazzara, S. Milioto, J. Phys. Chem. C 115 (2011) 20491-20498.

[13] E. Abdullayev, K. Sakakibara, K. Okamoto, W. Wei, K. Ariga, Y. Lvov, ACS Appl. Mater. Interfaces 3 (2011) 4040-4046.

[14] W.O. Yah, A. Takahara, Y.M. Lvov, J. Am. Chem. Soc. 134 (2011) 1853-1859.

[15] Z. Luo, H. Song, X. Feng, M. Run, H. Cui, L. Wu, et al., Langmuir 29 (2013) 12358-12366.

[16] P.J. Crowley, Pestic. Sci., in: M. Hudlicky, A. E. Pavlath (Eds.), American Chemical Society, Washington DC, 1995, vol. 53, 1998, pp. 263-263.

[17] V. Ćrkva, R. Polák, O. Paleta, K. Kefurt, J. Moravcová, M. Kodrček, et al., Carbohydr. Res. 339 (2004) 2177-2185.

[18] G. Cavallaro, G. Lazzara, S. Milioto, Langmuir 27 (2011) 1158-1167.

[19] L. Nordstierna, I. Furó, P. Stilbs, Langmuir 22 (2006) 7969-7974.

[20] S. Milioto, R. Crisantino, R.D. Lisi, A. Inglese, Langmuir 11 (1995) 718-724.

[21] A. Soto, A. Arce, M.K. Khoshkbarchi, Biophys. Chem. 74 (1998) 165-173.

[22] H. Freundlich, A.D. Jones, J. Phys. Chem. 40 (1935) 1217-1236.

[23] A.M. Wierenga, A.P. Philipse, Langmuir 13 (1997) 4574-4582.

[24] B.V. Derjaguin, L.D. Landau, Acta Physicochim. 14 (1941) 733-762.

[25] R. De Lisi, G. Lazzara, S. Milioto, N. Muratore, Phys. Chem. Chem. Phys. 5 (2003) 5084-5090. 